\title{
Methods and novel technology for microRNA quantification in colorectal cancer screening
}

\author{
Laura Moody ${ }^{1}$, Hongshan He ${ }^{4}$, Yuan-Xiang Pan ${ }^{1,2,3}$ and Hong Chen ${ }^{1,2^{*}}$ (D)
}

\begin{abstract}
The screening and diagnosis of colorectal cancer (CRC) currently relies heavily on invasive endoscopic techniques as well as imaging and antigen detection tools. More accessible and reliable biomarkers are necessary for early detection in order to expedite treatment and improve patient outcomes. Recent studies have indicated that levels of specific microRNA (miRNA) are altered in CRC; however, measuring miRNA in biological samples has proven difficult, given the complicated and lengthy PCR-based procedures used by most laboratories. In this manuscript, we examine the potential of miRNA as CRC biomarkers, summarize the methods that have commonly been employed to quantify miRNA, and focus on novel strategies that can improve or replace existing technology for feasible implementation in a clinical setting. These include isothermal amplification techniques that can potentially eliminate the need for specialized thermocycling equipment. Additionally, we propose the use of near-infrared (NIR) probes which can minimize autofluorescence and photobleaching and streamline quantification without tedious sample processing. We suggest that novel miRNA quantification tools will be necessary to encourage new discoveries and facilitate their translation to clinical practice.
\end{abstract}

Keywords: Colorectal cancer, Isothermal, Near-infrared (NIR), miRNA, Diagnosis, Optical imaging, Ytterbium

\section{Background}

Colorectal cancer (CRC) is one of the most common types of human cancers with high cancer-related morbidity and mortality rates. Low survival rates among metastatic CRC patients highlight the need for early detection in order to keep the tumor localized and dramatically improve prognosis [1]. In the early stages of the disease, CRC presents with the formation of benign polyps, or adenomas, in the colorectal mucosa. If found early, surgical intervention can remove adenomas before they become cancerous, but if untreated, adenomas can advance into invasive tumors and metastasize to the lymph nodes and other organs. Patients with cancer

\footnotetext{
* Correspondence: hongchen@illinois.edu

Supported by the USDA Cooperative State Research, Education and Extension Service, project number \#ILLU-971-344, a 20/20 grant from DNS, and CSTAR program at UIUC.

${ }^{1}$ Division of Nutritional Sciences, University of Illinois at Urbana-Champaign, 472 Bevier Hall, MC-182, 905 South Goodwin Avenue, Urbana, IL 61801, USA ${ }^{2}$ Department of Food Science and Human Nutrition, University of Illinois at Urbana-Champaign, 472 Bevier Hall, MC-182, 905 South Goodwin Avenue, Urbana, IL 61801, USA

Full list of author information is available at the end of the article
}

metastases only have a $10 \% 5$-year survival rate, but intervention during the early stages of the disease improves the survival rate to $90 \%$ [2]. The progression from adenoma to cancer can take 10-15 years, allowing for ample time to detect colon abnormalities [3]. However, current screening tools are primarily invasive and require specialized equipment. Thus, identification of effective noninvasive screening measures has tremendous potential to increase survivorship.

\section{Current CRC screening tools: a brief overview}

Currently, endoscopic procedures are the primary means of colorectal cancer screening. Colonoscopy is an invasive procedure that has been used for decades to visualize the entire colon and is recommended every 10 years for individuals over the age of 50 [4]. Numerous studies have established colonoscopy as a valuable screening tool that decreases CRC incidence up to $76 \%$ and lowers mortality by up to $65 \%$ [5-7]. The sensitivity of colonoscopies for colorectal adenomas ranges from 75 to $93 \%$ [4] while specificity approaches $100 \%$ [8]. A 
less invasive but less thorough alternative to colonoscopy is sigmoidoscopy in which only the lower part of the colon is examined. Sigmoidoscopy offers a sensitivity of 77 to $84 \%$ and a specificity of around $84 \%[9,10]$. Several studies have shown that sigmoidoscopy also decreases rates of CRC onset [11, 12]. One group showed that in patients screened with sigmoidoscopy, CRC development was reduced by $23 \%$ compared to unscreened controls and mortality was decreased by $31 \%$ [13]. While compliance in this study was high (71\%), other reports indicate that only $50 \%$ of the general population adheres to screening recommendations $[14,15]$. Because of its invasive nature, patients often report discomfort or pain during the procedure, which may prevent full compliance. Although rare, endoscopy also poses a risk for complications such as intestinal perforation and bleeding (Table 1) [4].

Several non-invasive alternatives to endoscopy have been proposed, but all are limited in sensitivity and specificity. The guaiac-based fecal occult blood test (FOBT) was developed to measure fecal blood in an inexpensive, noninvasive manner. Although the FOBT has been shown to reduce incidence and mortality of CRC $[2,5]$, the test is hindered by its insensitivity. False positives are common due to usage of mediations such as nonsteroidal anti-inflammatory drugs as well as dietary intake of red meat $[16,17]$. A single FOBT has a sensitivity of only around $50 \%[18,19]$, and while repeated testing can boost sensitivity, patients may be less willing to attend multiple appointments [20]. The fecal immunochemical test (FIT) is an antibody-based measurement of hemoglobin protein in stool. One study found that the FOBT provided a specificity of only $78 \%$ while the FIT offered a specificity of over 90\% [19]. The FIT also has a higher average sensitivity for CRC of $93 \%$, but has only a $48 \%$ sensitivity for advanced neoplasia, suggesting that it may not be optimal for identifying individuals at risk for CRC [21].

In 2014, the multitarget DNA test Cologuard ${ }^{\circ}$ was FDA-approved and made commercially available in the USA. In addition to fecal hemoglobin, Cologuard ${ }^{\circ}$ assays DNA methylation in the bone morphogenetic protein 3 (BMP3) and NDRG family member 4 (NDRG4) promoters and mutations in the KRAS proto-oncogene (KRAS) gene. The assay was reported to detect CRC with a sensitivity of $92 \%$ and premalignant lesions with a sensitivity of $42 \%$ [22]. In a screening of over 400 asymptomatic adults, methylation of BMP3 showed greater specificity for polyp detection than the FIT [23]. Another study in over 1000 subjects found that Cologuard ${ }^{\circ}$ detected CRC with $90 \%$ specificity and $98 \%$ sensitivity [24]. It was further noted that Cologuard ${ }^{\circ}$ could detect precancerous lesions with a sensitivity of $57 \%$ for precursors $\geq 1 \mathrm{~cm}$ and $83 \%$ for precursors $>3 \mathrm{~cm}$. This evidence

Table 1 Current CRC screening methods

\begin{tabular}{|c|c|c|c|c|c|c|}
\hline Tool & Blood or stool & Sensitivity (\%) & Specificity (\%) & Advantages & Limitations & Citation \\
\hline Colonoscopy & Invasive & $75-93$ & 100 & $\begin{array}{l}\text { - Well validated and } \\
\text { widely accepted } \\
\text { - High sensitivity and } \\
\text { specificity }\end{array}$ & $\begin{array}{l}\text { - Requires expertise to } \\
\text { perform/interpret } \\
\text { - Invasive } \\
\text { - Low compliance } \\
\text { - Risk of intestinal perforation } \\
\text { and bleeding }\end{array}$ & {$[4,8]$} \\
\hline Sigmoidoscopy & Invasive & $77-84$ & 84 & $\begin{array}{l}\text { - Typically does not } \\
\text { require sedation } \\
\text { - Less extensive bowel } \\
\text { preparation }\end{array}$ & $\begin{array}{l}\text { - Requires expertise to } \\
\text { perform/interpret } \\
\text { - Invasive } \\
\text { - Risk of intestinal perforation } \\
\text { and bleeding } \\
\text { - Not as thorough as } \\
\text { colonoscopy }\end{array}$ & {$[9,10]$} \\
\hline Fecal occult blood test (FOBT) & Stool & 50 & $91-98$ & $\begin{array}{l}\text { - Inexpensive } \\
\text { - Can be performed at } \\
\text { home }\end{array}$ & $\begin{array}{l}\text { - Low sensitivity } \\
\text { - Requires repeated testing }\end{array}$ & {$[18,19]$} \\
\hline Fecal immunochemical test (FIT) & Stool & 93 & 90 & $\begin{array}{l}\text { - Inexpensive } \\
\text { - Can be performed at } \\
\text { home } \\
\text { - High sensitivity }\end{array}$ & $\begin{array}{l}\text { - Not as sensitive to } \\
\text { colorectal neoplasia }\end{array}$ & {$[19,21]$} \\
\hline Cologuard & Stool & $92-98$ & 90 & $\begin{array}{l}\text { - Inexpensive } \\
\text { - Can be performed at } \\
\text { home }\end{array}$ & $\begin{array}{l}\text { - Not as sensitive to } \\
\text { colorectal neoplasia }\end{array}$ & {$[22,24]$} \\
\hline Carcinoembryonic antigen (CEA) & Blood & $74-80$ & 70-95 & - Easy to perform & $\begin{array}{l}\text { - Cannot detect early } \\
\text { stage CRC } \\
\text { - No standardized cutoff } \\
\text { values }\end{array}$ & {$[27,29]$} \\
\hline Epi proColon & Blood & $66-68$ & 91 & - Easy to perform & - Low sensitivity & {$[31,32]$} \\
\hline
\end{tabular}


suggests that fecal genetic markers can provide a viable means of cancer detection.

Like fecal screening, blood-based CRC biomarkers are not common and are limited in their ability to detect atrisk individuals. The carcinoembryonic antigen (CEA) is a glycoprotein associated with various cancers as well as gastrointestinal and liver diseases. CEA is a blood-based biomarker for CRC detection, although the sensitivity and specificity for early-stage CRC detection are relatively low $[25,26]$. Indeed, the sensitivity and specificity of CEA for detecting CRC up to 1 year before clinical presentation is only 57.5 and $81 \%$, respectively [27]. Elevated CEA levels in serum are correlated with higher CRC mortality rates [28]; however, early stages of CRC do not necessarily display high levels of CEA, and therefore, CEA is not widely accepted as a reliable diagnostic measure $[29,30]$.

In 2016, the first blood-based colorectal cancer screening test was approved by the FDA. Epi proColon ${ }^{\circ}$ measures methylated Septin9 (SEPT9) and was demonstrated to have a sensitivity of $68 \%$ [31]. Meta-analysis of 9870 subjects showed that methylated SEPT9 had a $66 \%$ sensitivity and $91 \%$ specificity for CRC [32]. The analysis suggests that these values are comparable to those provided by the FOBT, and thus, the authors propose Epi proColon ${ }^{\circ}$ as a complementary screening tool. Although not as sensitive as endoscopy, Epi proColon ${ }^{\circ}$ highlights the potential of blood-based epigenetic assays in CRC screening.

\section{miRNA in CRC}

miRNAs are small RNAs that regulate the expression of mRNA expression by binding to the 3' untranslated region (UTR) and altering ribosomal interactions, decapping or deadenylating the mRNA, or recruiting the RNA-induced silencing complex (RISC) [33]. miRNAs are involved in human carcinogenesis and alter expression of oncogenes and tumor suppressor genes as well as disrupt cellular functions such as stress response and apoptosis [34]. Tumor growth and metastasis in various cancer types can be mediated by dysfunctional regulation by miRNA [35-38].

Not only do tumors display aberrant miRNA expression, but biological fluids also show altered miRNA levels in cancer. Recent evidence suggests that miRNA may be excreted into circulation via exosomes and microvesicles or bound to proteins such as Argonaute or high-density lipoprotein (HDL) [39]. Indeed, several studies have demonstrated unique circulating miRNA profiles due to a wide variety of diseases, dietary patterns, and lifestyle factors [40-42]. Unlike in other cancers, fecal samples may potentially be used for patient screening in CRC. miRNA from tumor cells that slough off the lumen wall can be isolated in stool for quantification. Table 2 highlights the utility of noninvasive miRNA biomarkers for detecting CRC and identifying at-risk individuals.

\section{Circulating miRNA}

Circulating miRNA may serve as a reflection of the underlying disease in CRC [43-45]. Several plasma miRNA have been shown to be dysregulated in CRC (Table 2) [46-57]. miR-92 was first reported as a possible noninvasive biomarker for CRC diagnosis in 2009 $[58,59]$. Since then, a recent meta-analysis of over 500 colorectal cancer patients reported that miR-92a had a diagnostic sensitivity of $76 \%$ and sensitivity of specificity of $64 \%$ [60]. When several miRNAs (let-7g, miR-21, miR-92a, miR-181b, and miR-203) in serum were used as a biomarker profile panel for CRC diagnosis, sensitivity and specificity increased to 93 and $91 \%$, respectively. The same serum samples showed only 35\% sensitivity and $23 \%$ specificity for CRC when CEA and CA19-9 were used [61]. In addition to distinguishing normal individuals from cancer patients, circulating miRNA profiles have been shown to differ between healthy controls and patients with pre-cancerous adenomas [62]. High-throughput sequencing has facilitated the discovery of many more circulating miRNA that are differentially expressed in CRC [63, 64]; however, no blood-based miRNA tests are currently being used to screen for CRC.

\section{Fecal miRNA}

The FOBT and FIT are the primary stool-based screening tools for CRC, but the sensitivity and specificity are relatively low, especially at early pre-malignant time points [65]. Studies have reported that miR-92a [66], miR-20a [67], miR-21 [66], miR-221 [68], miR-18a [68], and miR-144* [69] are differentially expressed in CRC patients in comparison to healthy volunteers. These studies report sensitivities ranging from 55 to $74 \%$ and specificities ranging from 68 to $87 \%$. Table 2 demonstrates the efficiency of each fecal miRNA as a CRC biomarker. A majority of the studies investigating fecal miRNA are exploratory and are conducted on relatively small sample sizes. As more significant fecal miRNA biomarkers surface, adequate measurement tools will become more important to ensure reliable quantification.

\section{Conventional miRNA quantification tools}

While basic science and clinical trials have demonstrated a role for miRNA in colorectal cancer, the feasibility of clinical implementation remains questionable. Not only are sensitivity and reproducibility important characteristics of cancer biomarkers, but time, cost, and complexity of sample processing are crucial in facilitating the transition from the laboratory to the clinic. Currently, 
Table 2 Potential miRNA biomarkers for CRC screening

\begin{tabular}{|c|c|c|c|c|}
\hline miRNA & Blood or stool & Sensitivity (\%) & Specificity (\%) & Citation \\
\hline miR-92a & Blood and stool & $\begin{array}{l}72-79 \text { (blood) } \\
72 \text { (stool) }\end{array}$ & $\begin{array}{l}59-69 \text { (blood) } \\
73 \text { (stool) }\end{array}$ & {$[60,66]$} \\
\hline miR-20a & Blood and stool & $\begin{array}{l}46 \text { (blood) } \\
55 \text { (stool) }\end{array}$ & $\begin{array}{l}73 \text { (blood) } \\
82 \text { (stool) }\end{array}$ & {$[46,67]$} \\
\hline miR-21 & Blood and stool & $\begin{array}{l}62-85 \text { (blood) } \\
56 \text { (stool) }\end{array}$ & $\begin{array}{l}79-88 \text { (blood) } \\
73 \text { (stool) }\end{array}$ & {$[52,55,66$} \\
\hline miR-221 & Blood and stool & $\begin{array}{l}86 \text { (blood) } \\
62 \text { (stool) }\end{array}$ & $\begin{array}{l}41 \text { (blood) } \\
74 \text { (stool) }\end{array}$ & {$[51,68]$} \\
\hline miR-18a & Stool & 61 & 69 & [68] \\
\hline miR-135b & Stool & 78 & 68 & [66] \\
\hline miR-144* & Stool & 74 & 87 & [67] \\
\hline miR-199a-3p & Blood & 48 & 75 & [50] \\
\hline miR-155 & Blood & 58 & 95 & [48] \\
\hline miR-183 & Blood & 74 & 89 & {$[56]$} \\
\hline miR-29a & Blood & $53-65$ & $85-93$ & [57] \\
\hline miR-29b & Blood & 61 & 73 & [47] \\
\hline miR-210 & Blood & 75 & 74 & [53] \\
\hline miR-196b & Blood & 63 & 87 & [54] \\
\hline miR-139-3p & Blood & 97 & 98 & [49] \\
\hline miR-622 & Blood & 88 & 64 & [49] \\
\hline miR-506 & Blood & 61 & 77 & [72] \\
\hline miR-4316 & Blood & 75 & 77 & [72] \\
\hline
\end{tabular}

quantitative real-time PCR (qPCR), microarray, and next-generation sequencing (NGS) are the most commonly used techniques for quantifying miRNA. While such procedures are routinely used in the laboratory, not one is completely ideal for rapid, reproducible miRNA detection. Here, we outline the chemistry involved in conventional quantification tools. We draw attention to the advantages of each platform and discuss areas where each falls short (Table 3).

\section{$q P C R$}

qPCR is a means of measuring single miRNAs and is currently the gold standard for miRNA quantification, and it has been routinely utilized for measuring miRNA in both blood [70-72] and stool [66, 69, 73] samples in order to explore miRNA for CRC screening. Typically, after miRNA is isolated, it is amplified with reverse transcription (RT)-PCR to make cDNA. The sequence of interest is then amplified and measured in real time using fluorescent probes. SYBR Green and Taqman chemistry are the two primary systems used for fluorescent detection. SYBR Green binds double-stranded DNA and fluoresces as the target sequence is amplified. This eliminates the need for separate probes for each miRNA of interest, but reduces the assay's specificity, as all double-stranded DNA fragments produce fluorescence. Taqman technology relies on a labeled probe. During the
qPCR reaction, the labeled probe binds to the target sequence. The primers anneal and are extended by Taq polymerase, which degrades the probe and releases the fluorophore. While Taqman provides superior miRNA detection sensitivity and specificity, labeled probes must be synthesized for every region of interest, which is less cost-effective when quantifying numerous genes.

miRNA differs from mRNA in that sequences are short ( $22 \mathrm{nt})$ and are generally less abundant. Thus, qPCR procedures must avoid primer dimers and ensure a low detection threshold. Currently, two methods attempt to overcome these hurdles. The use of stem-loop RT primers has been shown to improve miRNA detection sensitivity and specificity over linear primers, potentially via spatial constraints, base-stacking, and increased thermal stability [74]. However, in order to enrich the assay for the miRNA of interest, this method requires separate RT primers for each miRNA. Alternatively, Exiqon has developed a locked nucleic acid (LNA) assay in which the RT-PCR reaction needs only to be performed once. The RT reaction is performed with a universal mature miRNA primer, and for qPCR, LNA primers may be used with SYBR Green for amplification. LNAs have a higher affinity to complementary bases. Therefore, shorter sequences may be synthesized to prevent primer overlap. While the LNA-based technique has efficiencies comparable to those of stem-loop primers, measurements 
Table 3 miRNA quantification technology

\begin{tabular}{|c|c|c|c|}
\hline Technology & Advantages & Limitations & Citations \\
\hline$\overline{\mathrm{qPCR}}$ & $\begin{array}{l}\text { - Current gold standard for sensitivity } \\
\text { and specificity }\end{array}$ & - No genome-wide coverage & [74-76] \\
\hline Microarray & $\begin{array}{l}\text { - Commercially available reagents } \\
\text { - Genome-wide coverage }\end{array}$ & $\begin{array}{l}\text { - Specific probes } \\
\text { - Specialized equipment } \\
\text { - Lack of reproducibility between } \\
\text { platforms } \\
\text { - Difficult data normalization }\end{array}$ & [81-87] \\
\hline NGS & $\begin{array}{l}\text { - Genome-wide coverage } \\
\text { - Multiple samples may be run in parallel } \\
\text { - Promotes novel miRNA discovery } \\
\text { - Can detect polymorphisms }\end{array}$ & $\begin{array}{l}\text { - Complicated, non-standardized } \\
\text { data analysis }\end{array}$ & [93-97] \\
\hline Isothermal amplification & $\begin{array}{l}\text { - No need for thermocycling equipment } \\
\text { - Can improve existing qPCR, microarray, } \\
\text { and NGS methods }\end{array}$ & $\begin{array}{l}\text { - Disadvantages are technique- } \\
\text { specific (see below) }\end{array}$ & [101] \\
\hline - Exponential amplification & - High sensitivity & $\begin{array}{l}\text { - May require a nicking enzyme, } \\
\text { which complicates primer design }\end{array}$ & {$[102,103]$} \\
\hline - Rolling circle amplification & $\begin{array}{l}\text { - } 1 \text { primer } \\
\text { - Can be optimized for linear or } \\
\text { exponential amplification }\end{array}$ & $\begin{array}{l}\text { - Requires } 2 \text { enzymes (polymerase } \\
\text { and ligase) } \\
\text { - Initial denaturation not performed } \\
\text { at room temperature }\end{array}$ & [105-109] \\
\hline $\begin{array}{l}\text { - Duplex-specific nuclease signal } \\
\text { amplification }\end{array}$ & - High specificity & - Enzyme is not readily available & [110-113] \\
\hline - Hybridization chain reaction & - No polymerase & - Linear amplification only & [114-120] \\
\hline Near-infrared technology & $\begin{array}{l}\text { - No autofluoresence } \\
\text { - Minimal photobleaching } \\
\text { - No tedious treatment of sample before } \\
\text { or after the test }\end{array}$ & $\begin{array}{l}\text { - Lanthanide probes are not yet } \\
\text { commercially available and must } \\
\text { be optimized }\end{array}$ & {$[132,140-143]$} \\
\hline
\end{tabular}

are more variable, less efficient, and specificity may be an issue $[75,76]$.

\section{Microarray}

In order to identify novel biomarkers for CRC screening, microarray has been extensively used to quantify all human miRNA in both blood [77-79] and fecal samples $[67,80]$. Microarray is a hybridization technique that utilizes DNA probes to quantify specific miRNAs. Much like in qPCR, isolated RNA is amplified by RT-PCR. Biotinylation and fragmentation are performed and the sample is incubated over several hours during which the sample hybridizes with probes that are fixed on the microarray surface. The microarray cartridge is then washed to remove non-specific binding. Finally, the plate is stained with streptavidin bound to a fluorophore. Streptavidin binds to the biotinylated sequences and the fluorophore can be excited by a laser.

Several microarray platforms have been adapted for miRNA quantification, including GeneChip (Affymetrix), miRCURY LNA (Exiqon), and SurePrint (Agilent). All platforms have designed probes specific for mature miRNA sequences, but major differences include hybridization and washing procedures as well as fluorescent dyes. Microarray technology can be used to measure multiple miRNA simultaneously, but several drawbacks limit their utility in the clinic. Specific probes, specialized equipment, and differences in hybridization procedures drive up cost and compromise reproducibility between platforms [81, 82]. Furthermore, data normalization is difficult and time-consuming, and no single method has been universally accepted to analyze microarray data [83-86]. miRNA poses an even greater challenge for normalization due to the small number of miRNA and weak expression levels [87].

\section{Next-generation sequencing}

Massively parallel next-generation sequencing (NGS) was first introduced in 2005 and has since enabled researchers to measure miRNA in a genome-wide fashion [88]. Several platforms are available from Life Technologies, Illumina, and others. The typical workflow involves RNA isolation, library preparation, sequencing, and data analysis. Library construction involves $5^{\prime}$ and $3^{\prime}$ adapter ligation and amplification. Adapters are platformspecific and provide a bar code that is recognized during amplification. Amplification has traditionally been performed using either emulsion PCR (emPCR) or bridge PCR (bPCR). The Sequencing by Oligonucleotide Ligation and Detection (SOLiD) platform developed by Life Technologies has employed emPCR. In emPCR, cDNA is captured on streptavidin beads which are placed in an oil emulsion mixture, creating separate water compartments containing only one template [89]. 
This partitioning minimizes hybridization between amplification products. Life Technologies has also introduced an isothermal template walking approach for library construction [90]. Bar-coded human exome fragment libraries are modified to contain 3 ' poly-T overhangs. Solid-phase primers capture the nicked template DNA and are extended at room temperature with Bst polymerase. Next, isothermal strand displacement was run with Bst and a solution-phase primer. Compared to emPCR or bPCR, this template walking method does not use microbeads and is more cost-effective. Conversely, Illumina sequencing uses isothermal solid-phase bPCR. In this method, two oligos are situated on a glass slide. The template hybridizes with the first oligo, is elongated by a polymerase, and is washed away. The single-stranded sequence then bends over and hybridizes with a second oligo and a double-stranded bridge is created by the polymerase. The process is repeated until the cDNA has been sufficiently amplified [90].

Once a library is generated, sequencing may be performed. In the SOLiD platform, fluorescently labeled dinucleotide sequences hybridize with complementary template sequences and are ligated, and fluorescence is measured [91]. Illumina platforms utilize sequencing-bysynthesis, which involves adding fluorescently labeled nucleotides to a nucleotide chain [92]. After the addition of each nucleotide, the fluorescent signal is captured. Next, data analysis is performed, including sequence alignment to a reference genome, count generation, data pre-processing, and statistical analysis [93].

Compared to the preceding Sanger sequencing technology, NGS is not limited by the use of gel or polymer separation media and thus allows multiple samples to be run in parallel [94]. Given its ability to query the entire genome, NGS is ideal for novel biomarker discovery. As the cost continues to decrease, NGS is quickly becoming the preferred genome-wide quantification method [95-97]. However, implementing such technology in the clinic poses serious challenges. Like microarray, NGS data analysis is complicated and not standardized [93]. Several studies have used NGS in blood samples in order to identify candidate miRNA for CRC screening [98-100], but these studies are often validated using another more targeted approach (e.g., qPCR).

\section{Novel miRNA quantification tools Isothermal amplification}

All of the aforementioned methods must amplify genetic material in order to detect signal. While NGS employs isothermal bPCR and template walking, qPCR and microarray traditionally utilize thermocycling to create cDNA. This involves a denaturation step followed by several rounds of primer annealing and elongation by a Taq polymerase. By contrast, isothermal reactions are performed at a constant temperature. Several variations of isothermal amplification exist, including exponential amplification reactions, loop-mediated amplification, rolling circle amplification, duplex-specific nuclease signal amplification, and hybridization chain reaction [101].

Exponential amplification reactions employ a nicking enzyme to catalyze miRNA amplification. Jia et al. used an amplification template with a nicking enzyme recognition sequence flanked by two identical sequences that are complementary to the target miRNA [102]. Once the miRNA of interest hybridizes with the amplification template, it is extended by Vent (exo-) DNA polymerase, then cleaved by the nicking enzyme (Fig. 1a). The released product then serves as the trigger for the next

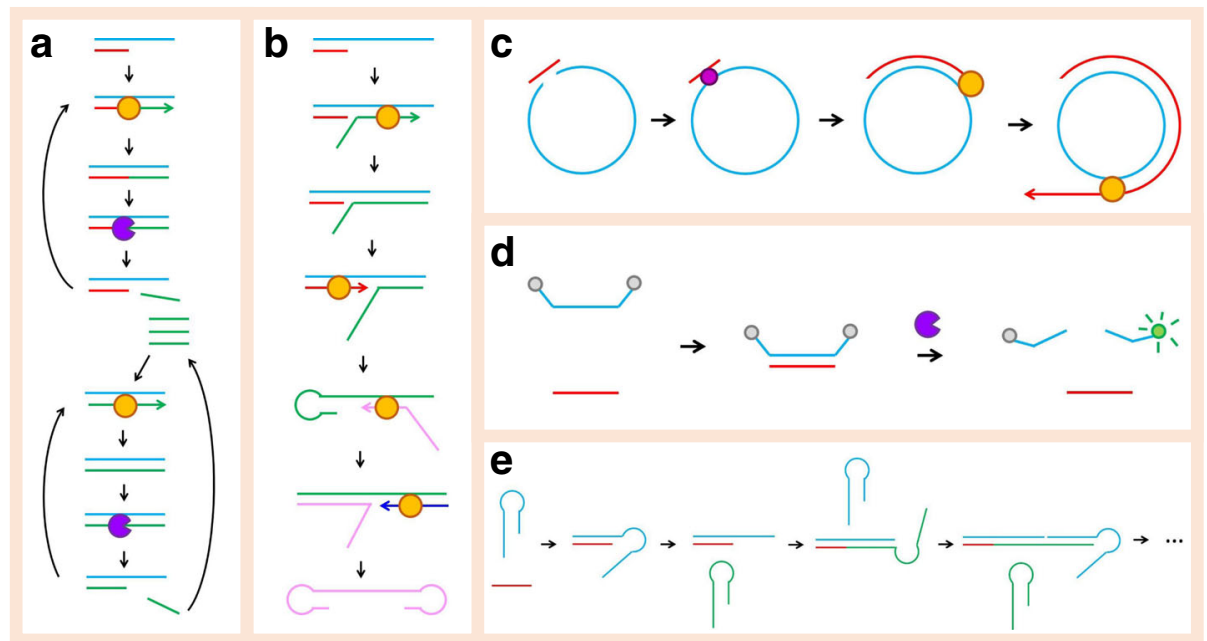

Fig. 1 Summary of isothermal amplification techniques. a Exponential amplification using a nicking enzyme. b Exponential amplification without nicking enzyme. c Rolling circle amplification. $\mathbf{d}$ Duplex-specific nuclease amplification. e Hybridization chain reaction 
amplification reaction. Using a SYBR Green dye, the product can be detected in real time. This procedure has been further modified using stem-looped amplification template and strand displacement, which eliminated the need for a nicking enzyme (Fig. 1b) [103]. In this design, an amplification template, forward inner primer, backward inner primer, and outer primer are required. At the $5^{\prime}$ end, the template contains a binding region for the outer primer, and at the $3^{\prime}$ end of the template, there is a sequence complementary to the target miRNA. In between the two ends, there are binding regions for the backward and forward inner primers. First, the forward inner primer binds the amplification template and is extended by Bst DNA polymerase. Next, the miRNA binds and is extended to displace the forward inner primer, creating a $5^{\prime}$ stem-loop from the forward inner primer. At the 3' end of the stem-loop forward inner primer, the backward inner primer is elongated with Bst DNA polymerase and displaced by the backward outer primer, allowing the formation of the $5^{\prime}$ stem loop. SYBR Green can again be used to quantify the amplification product. These methods have been shown to have extremely high miRNA detection sensitivity, capturing signal at miRNA levels as low as $10^{-10} \mathrm{pmol}$ [103]. However, primer design is complicated and if a nicking enzyme is used, the template must contain a recognition site.

Rolling circle amplification provides another isothermic technique for miRNA quantification. In this method, the miRNA of interest ligates with a circular ssDNA template and is extended with DNA polymerase (Fig. 1c). Once the full length of the template has been elongated, the product is displaced and the polymerase continues around the circular primer, creating many copies of the miRNA. This method was first used for miRNA by Jonstrup et al. in 2006 but has since been improved [104]. Modifications to the original method include the use of a hairpin DNA probe to trigger the rolling circle reaction $[105,106]$ and immediate miRNA quantification via an enzymatic luminescence assay [107] or a fluorescently labeled probe [108]. Solid-phase rolling circle amplification was also applied for NGS amplification by Drmanac et al. to avoid the need for precise template concentrations [109]. Rolling circle amplification is simple in that only one primer is necessary and may be optimized to perform either linear or exponential amplification. However, two enzymes are required, both a polymerase and a ligase, and the initial denaturation is not performed at room temperature.

Duplex-specific nuclease signal amplification employs a duplex-specific nuclease that specifically cleaves double-stranded DNA or DNA:RNA heteroduplexes. The use of duplex-specific nuclease signal amplification for miRNA detection was first introduced by Yin et al. [110]. In their design, a Taqman probe hybridizes with the miRNA of interest and the probe is digested by the duplex-specific nuclease, releasing a fluorescent signal (Fig. 1d). This method has been paired with $\mathrm{Fe}_{3} \mathrm{O}_{4} @ \mathrm{Ag}$ nanoparticles [111], $\mathrm{Au}$ nanoparticles [112], and $\mathrm{Au}$ nanoparticles bound to $\mathrm{MoS}_{2}$ microcubes [113] for signal amplification. The duplex-specific nuclease is very effective at increasing assay specificity in that it selectively digests dsDNA and heteroduplexes but leaves ssDNA and imperfectly matched sequences untouched. However, the enzyme itself is isolated from the Kamchatka crab and is currently not widely available. The technique also results in linear, rather than exponential amplification which is not ideal for detection of weakly expressed miRNA.

Hybridization chain reaction is a method in which two DNA hairpin primers undergo a series of hybridization reactions to create a self-assembled DNA nanostructure (Fig. 1e) [114]. The reaction is initiated by a ssDNA that hybridizes with the sticky end of the first primer and opens the hairpin via strand displacement, creating a sticky end. That stick end opens the second hairpin primer and exposes a new sticky end that contains a sequence identical to the initiator, which can act on the first primer. This cycle continues, forming a nicked double helix. When a miRNA is used as the initiator, this method can be used for miRNA quantification and visualization. Signal detection has been facilitated through the use of $\mathrm{Ag}$ [115] and $\mathrm{Au}$ [116] nanoparticles, as well as fluorescently labeled primers that are stabilized by a tetrahedral DNA scaffold [117] or a graphene oxide surface [118]. Recently, $\mathrm{Bi}$ et al. coupled hybridization chain reaction with catalytic hairpin assembly, a method in which an initiator sequence catalyzes the self-assembly of hairpin primers to form complex nanostructures [119, 120]. Two DNA hairpins were used for catalytic assembly, and one unlabeled hairpin and one FAM-labeled hairpin were used for the hybridization chain reaction. When the miRNA of interest was introduced, it hybridized with the sticky end of the first primer and toehold-mediated strand displacement occurred as the first hairpin primer was opened. The exposed single-stranded sequence on the first primer then opened the second hairpin primer through an analogous reaction. The second primer then displaced the miRNA on the first primer, forming a branched DNA junction. Subsequently, the third and fourth hairpin primers catalyzed the hybridization chain reaction at each of the branches and the chemoluminescence signal was measured. The primary advantage of hybridization chain reaction is that it does not require a polymerase or any other enzyme, as it simply uses the potential energy of the hairpin primers. However, hybridization chain reaction only performs linear amplification and thus does not provide the same miRNA detection sensitivity as exponential amplification systems [114]. 
While isothermal amplification has not yet been used to quantify miRNA in CRC, it has been used to measure DNA and mRNA. Two investigations have used isothermal amplification to amplify DNA for microarray analysis of copy number variation. Cardoso et al. used Phi29 DNA polymerase and random hexamer primers for multiple strand displacement amplification with only $2 \mathrm{ng}$ of DNA [121]. A subchromosomal $5 q$ deletion was detected in tumor cells from patients with familial adenomatous polyposis (FAP). In another study, single primer isothermal amplification was used and a gain of $20 \mathrm{q}$ was found to be associated with tumor invasiveness in CRC patients [122]. Isothermal amplification of mRNA in CRC patients has been performed using loop-mediated exponential amplification [123, 124]. Higher levels of cytokeratin 19 (CK19) in lymph nodes was positively correlated with tumor size in stage I and II CRC patients.

The application of isothermal miRNA amplification in CRC screening has yet to be explored, but several other studies have highlighted the use of isothermal amplification in quantifying miRNA in other cancers. Persano et al. used a nicking enzyme amplification reaction to quantify higher levels of miR-10b in breast cancer cell lines and in serum of mice with breast tumors [125]. Catalytic hairpin assembly and rolling circle amplification were also used to detect high levels of miR-21 in breast cancer cell lines [126-128]. Additionally, rolling circle amplification was employed to measure high levels of miR-21 and miR-486-5p in lung cancer cell lines and in serum from lung cancer patients, respectively [106, 129]. Although isothermal amplification of miRNA is currently not a widespread methodology, it has tremendous potential to simplify screening across cancers.

\section{Near-infrared technology}

Traditional miRNA quantification tools require extensive sample preparation including miRNA isolation and amplification. While isothermal amplification has the potential to improve existing platforms by minimizing equipment demands and thus reducing cost, other technology has the potential to stand alone and possibly act as an alternative to commonly used techniques. Nearinfrared (NIR) imaging for detection of miRNA in biological samples would potentially eliminate the need for PCR and complicated sample processing. Furthermore, it confers numerous advantages over its optical imaging counterparts, such as minimal photobleaching and autofluorescence [130].

NIR-emitting lanthanoids can be utilized as probes. For instance, ytterbium (III) is one of the 14 elements in the periodic table that belong to the lanthanoid series. Similar to other members in the series, ytterbium (III) ion is quite stable under physiological conditions and is environmentally benign. One unique property of ytterbium
(III) ion is its narrow emission centered at $980 \mathrm{~nm}$ under illumination [131]. The emission is the result of an electronic transition from an inner $4 f$ shell; as a result, the emission wavelength is almost fixed and has little effect from the surrounding environment. Several other lanthanoid ions are also capable of emitting in the NIR region including the neodymium ion $(1060 \mathrm{~nm})$ and erbium ion (1540 nm); however, Zhong et al. have shown that ytterbium (III) outperforms others in terms of emission efficiency [132]. In the same experiment, ytterbium (III) was paired with a dye to further sensitize its emission. The dye molecule, upon excitation with a light source, transfers energy through its excited states (triplet or singlet states) to the ytterbium (III) ion. When the ytterbium (III) ion relaxes to its ground state, it produces characteristic emission. This process overcomes the requirement of a coherent and intense light source in direct excitation processes and is quite suitable and desirable for biomedical applications.

It should be noted that sensitization can be achieved either through a F rster resonance energy transfer (FRET) process or direct coordination of dye molecules to ytterbium (III) ion [133, 134]. Figure 2 illustrates the working principle of linear FRET probes using two antisense oligonucleotides. A donor chromophore and an acceptor chromophore are first labeled respectively on two oligonucleotides on the $3^{\prime}$ and 5 ' ends. Upon mixing with a test sample, they hybridize with target nucleic acid in adjacent regions. This brings the donor and the acceptor into close proximity. Under light illumination, the donor transfers energy to the acceptor to produce fluorescence for quantification or imaging of target nucleic acid levels.

Visible light-emitting lanthanoids including europium (III) and terbium (III) have been reported for DNA, RNA, and avidin detection. Karhunen et al. separately attached a europium (III) chelate (an acceptor) and a dye molecule (a donor) to biotins [135, 136]. After mixing with avidin, the strong interaction between the avidin and the biotin brings the donor and the acceptor in proximity to initiate the energy transfer for emission under UV light excitation [135]. A similar homogeneous assay was also used to detect DNA in which a dye and europium (III) chelate are labeled separately on complementary oligonucleotides of target nucleic acids [136]. Once they hybridize with target DNA, the dye coordinates to the europium (III) ion and sensitizes its emission. Abe et al. linked a europium (III) (or terbium (III)) chelate and a 7-amino-4-methyl-2 $(1 \mathrm{H})$-quinolinone to different oligonucleotides [137]. Under illumination, the quinolinone was triggered by a luminogenic agent and converted to the quinoline to sensitize the europium (III) for emission. The luminogenic probe could detect 


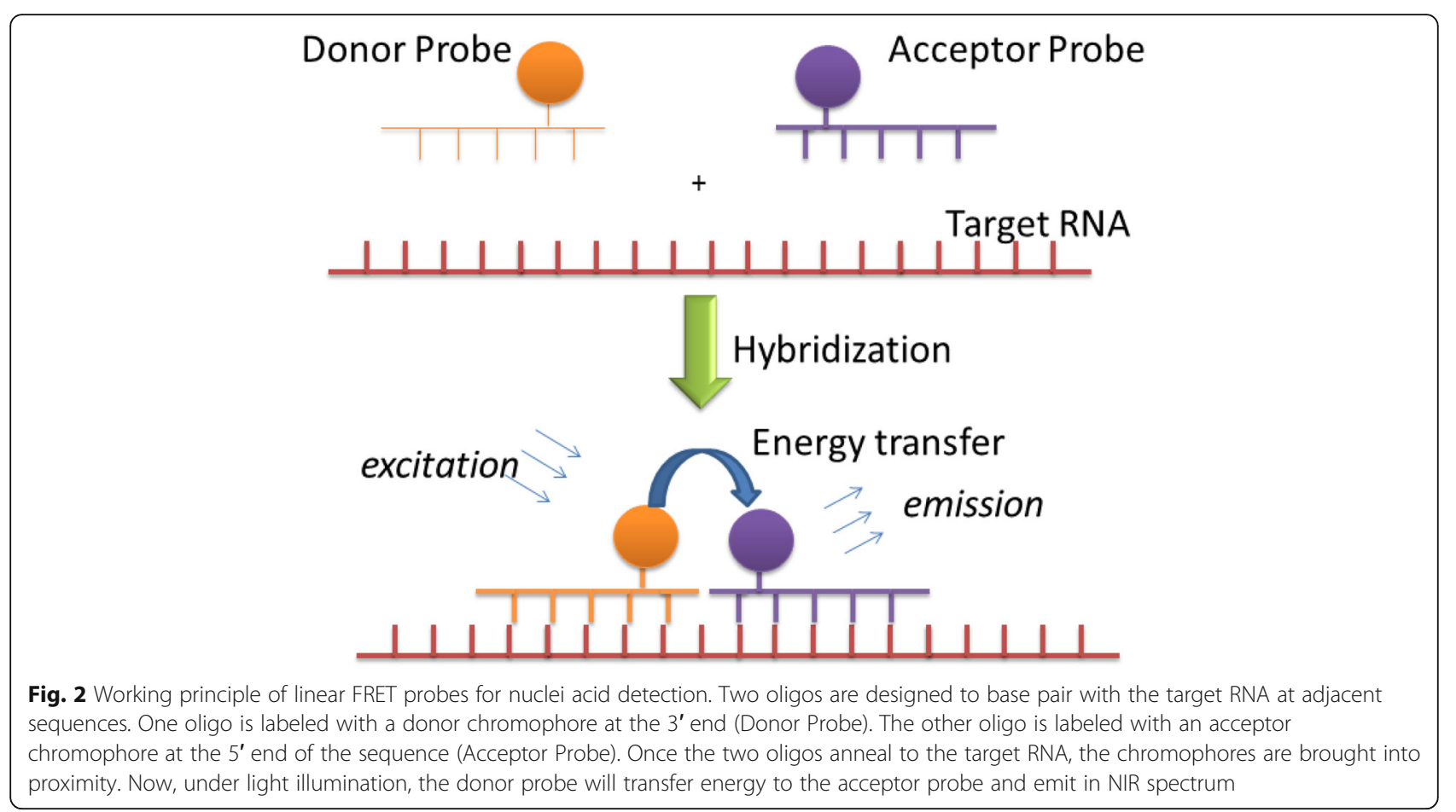

DNA and RNA in a crude solution of living bacterial cells with a signal to noise ratio about 400. Two significant disadvantages were observed in these studies: (1) signal intensity was dramatically compromised due to the use of a time-gated technique to reduce the autofluorescence since europium (III) and terbium (III) both emit in the visible region (green and red) and (2) severe photobleaching due to the use of short wavelength light source (UV) because the dye molecules did not absorb long wavelength light. Both limitations are undesirable for nucleic acid detection.

Several studies have used lanthanoid-based probes for upconversion and detection in the visible spectrum, but only a few have harnessed the technology for nucleic acid detection at NIR wavelengths. Nonat et al. used ytterbium in a cyclen-ruthenium(phen) ${ }_{3}$ (phen: 1,10-phenanthroline) to bind DNA and measured changes in the NIR emission spectrum at different titrations of DNA [138]. This method was only employed to detect the presence of DNA rather than specific sequences. More recently, the transition metals ruthenium and osmium were used in heterometallic complexes with a pyrenylbiimidazolate bridging ligand. It was again shown that the complex could act as a DNA intercalator for detection in the NIR spectrum [139]. NIR detection of miRNA has been accomplished through $\mathrm{Ag}_{2} \mathrm{~S}$ quantum dots [140] and DNA-conjugated Ag nanoclusters [141]. However, these methods either required the use of an electrode or did not provide comparable miRNA detection sensitivity to amplification-based strategies, both of which would be problematic when rapid detection of less abundant miRNA is necessary.

Boron-dipyrromethene (BODIPY)-coupled lanthanide probes may be especially desirable for miRNA detection [132, 142, 143]. Zhong et al. synthesized a BODIPY ligand with 8-hydroxylquinoline in its meso position (8HOQ-BODIPY) [132]. The ligand interacted with lanthanide ions, forming trisquinoline-like complexes that emitted in the NIR region upon visible light excitation. In a related experiment, the BODIPY dye was iodized with ytterbium (III) to form a probe with an empirical composition of $\left[\mathrm{Yb}(8-\mathrm{OQ}-\mathrm{BODIPY}-3 \mathrm{I})_{3}\right]$ [143]. The sensitization capability of the iodized $[\mathrm{Yb}(8-\mathrm{OQ}-\mathrm{BOD}-$ IPY-3I $)_{3}$ ] probe was tested and it displayed an emission lifetime of $\sim 95 \pm 17 \mu$ s. The emission efficiency was $4.75 \%$ (calculated from the equation $\Phi_{\mathrm{Yb}}=\tau_{\mathrm{obs}} / \tau_{0}$, $\tau_{0}=2 \mathrm{~ms}$ ) [132, 144]. Under $543 \mathrm{~nm}$ visible light excitation, the complex exhibited maximum emission intensity at $975 \mathrm{~nm}$. The results clearly demonstrate the ability and potential of BODIPY dyes to efficiently sensitize lanthanide ions for NIR emission.

Many optical probes are fluorescence-based molecules $[145,146],=$ and are widely used in a variety of medical diagnostic tests [147]. Several visible lights emitting lanthanoids including europium (III) and terbium (III) have been reported for DNA, RNA, and avidin detection [135-137]. The nucleic acid detection sensitivity of a probe relies upon the ratio of its fluorescent intensity 
and background signal. The significant overlap of biological substrate autofluorescence and fluorescence from the optical probes dramatically compromises detection sensitivity [148]. The NIR probe-based detection can overcome several drawbacks of current detection methods. First, it eliminates the autofluorescence. This is because nucleic acids only produce fluorescence in the visible region and do not produce emission in the NIR spectrum. By shifting the fluorescence of probes to the NIR region, the overlap that is quite significant in stateof-the-art probes will no longer exist. This creates "zero" background and increases the signal-to-noise $(\mathrm{S} / \mathrm{N})$ ratio of the detection dramatically [149]. Also, S/N benefits from weak Rayleigh scattering effect in the NIR region. Second, NIR can probes reduce photobleaching. Many commercially available probes require UV or near-UV light excitation, causing severe degradation of biological substrates. The proposed BODIPY-coupled NIR probes are efficiently excited by long wavelength light sources with much lower frequencies [143]. This prevents sample degradation due to photobleaching caused by higher energy light sources. Most importantly, the probes do not require tedious treatment of sample before and after the test. This not only expedites detection but also maintains the integrity of the sample for accurate detection of nucleic acids.

\section{Conclusion}

CRC is unique in that both blood and fecal samples can be used for biomarker discovery. However, non-invasive screening for colorectal cancer is currently not as sensitive or specific as widespread endoscopic methods. Genetic tests may provide a more sensitive screening for at-risk individuals. Indeed, commercially available Cologuard ${ }^{\circ}$ and Epi proColon ${ }^{\circ}$ detect aberrant DNA methylation, and several studies have suggested that miRNA profiles in stool and in circulation also undergo drastic changes during CRC pathogenesis. With breakthroughs in basic science, technology must be developed to meet clinical demands. Current microarray, sequencing, and PCR-based methods of miRNA quantification are costly, complex, and have high time and resource demands. We suggest that isothermal amplification and NIR imaging may overcome the shortcomings of current techniques by reducing time and cost, eliminating the need for specialized equipment, and ensuring sensitive and specific miRNA detection. The development of novel technology will expedite biomarker discovery and enable their clinical implementation for effective CRC screening.

\section{Abbreviations}

BODIPY: Boron-dipyrromethene; bPCR: Bridge PCR; CEA: Carcinoembryonic antigen; CRC: Colorectal cancer; CRT: Chemoradiation therapy; DNA: Deoxyribonucleic acid; emPCR: Emulsion PCR; FIT: Fecal immunochemical test; FOBT: Fecal occult blood test; FRET: Förster resonance energy transfer; LNA: Locked nucleic acid; miR (miRNA): Micro ribonucleic acid; NGS: Next-generation sequencing; NIR: Near-infrared; PCR: Polymerase chain reaction; qPCR: Quantitative real time PCR; RNA: Ribonucleic acid; RT: Reverse transcription; SOLiD: Sequencing by oligonucleotide ligation and detection

\section{Acknowledgements}

We thank the University of Illinois for their financial support regarding the publication fee.

Support of this research by the National Science Foundation under Award CHE-1507871 $(\mathrm{HH})$ is gratefully acknowledged.

\section{Funding}

Support for this work was provided by a Vision 20/20 grant from Division of Nutritional Sciences in College of Agricultural, Consumer, and Environmental Sciences and the Cancer Scholars for Translational and Applied Research (C*STAR) program funded by the University of Illinois and the Carle Cancer Center.

\section{Availability of data and materials}

Not applicable.

\section{Authors' contributions}

All authors were responsible for the draft of the work or critical revision for important intellectual content. All authors read and approved the final manuscript.

Ethics approval and consent to participate

Not applicable.

Consent for publication

Not applicable.

\section{Competing interests}

The authors declare that they have no competing interests.

\section{Publisher's Note}

Springer Nature remains neutral with regard to jurisdictional claims in published maps and institutional affiliations.

\section{Author details}

${ }^{1}$ Division of Nutritional Sciences, University of Illinois at Urbana-Champaign, 472 Bevier Hall, MC-182, 905 South Goodwin Avenue, Urbana, IL 61801, USA. ${ }^{2}$ Department of Food Science and Human Nutrition, University of Illinois at Urbana-Champaign, 472 Bevier Hall, MC-182, 905 South Goodwin Avenue, Urbana, IL 61801, USA. ${ }^{3}$ Illinois Informatics Institute, University of Illinois at Urbana-Champaign, Urbana, IL 61801, USA. Department of Chemistry, Eastern Illinois University, Charleston, IL 62910, USA.

Received: 21 July 2017 Accepted: 17 October 2017

Published online: 24 October 2017

\section{References}

1. Siegel RL, et al. Colorectal cancer statistics, 2017. CA Cancer J Clin. 2017;67(3):177-93.

2. Bretthauer M. Colorectal cancer screening. J Intern Med. 2011:270(2):87-98.

3. Dekker E, van Gulik T. Colorectal cancer: what the clinician wants to know. Cancer Imaging. 2005:5 Spec No A:S127-32.

4. Lin JS, et al. Screening for colorectal cancer: updated evidence report and systematic review for the US preventive services task force. JAMA. 2016; 315(23):2576-94.

5. Baxter NN, et al. Association between colonoscopy and colorectal cancer mortality in a US cohort according to site of cancer and colonoscopist specialty. J Clin Oncol. 2012;30(21):2664-9.

6. Kahi CJ, Anderson JC, Rex DK. Screening and surveillance for colorectal cancer: state of the art. Gastrointest Endosc. 2013;77(3):335-50.

7. Baxter NN, et al. Association of colonoscopy and death from colorectal cancer. Ann Intern Med. 2009;150(1):1-8.

8. Walleser $\mathrm{S}$, et al. What is the value of computered tomography colonography in patients screening positive for fecal occult blood? A systematic review and economic evaluation. Clin Gastroenterol Hepatol. 2007:5(12):1439-46. quiz 1368 
9. Sung JJ, et al. Screening for colorectal cancer in Chinese: comparison of fecal occult blood test, flexible sigmoidoscopy, and colonoscopy. Gastroenterology. 2003;124(3):608-14.

10. Graser A, et al. Comparison of $C T$ colonography, colonoscopy, sigmoidoscopy and faecal occult blood tests for the detection of advanced adenoma in an average risk population. Gut. 2009;58(2):241-8.

11. Neugut Al, Lebwohl B. Colonoscopy vs sigmoidoscopy screening: getting it right. JAMA. 2010;304(4):461-2.

12. Segnan $\mathrm{N}$, et al. Once-only sigmoidoscopy in colorectal cancer screening: follow-up findings of the Italian randomized controlled trial_-SCORE. J Natl Cancer Inst. 2011;103(17):1310-22.

13. Atkin WS, et al. Once-only flexible sigmoidoscopy screening in prevention of colorectal cancer: a multicentre randomised controlled trial. Lancet. 2010;375(9726):1624-33.

14. Yeazel MW, et al. Colorectal cancer screening adherence in a general population. Cancer Epidemiol Biomark Prev. 2004;13(4):654-7.

15. Subramanian $\mathrm{S}$, et al. Adherence with colorectal cancer screening guidelines: a review. Prev Med. 2004;38(5):536-50.

16. Narula $\mathrm{N}$, et al. Fecal occult blood testing as a diagnostic test in symptomatic patients is not useful: a retrospective chart review. Can J Gastroenterol Hepatol. 2014:28(8):421-6.

17. Levin $B$, et al. Screening and surveillance for the early detection of colorectal cancer and adenomatous polyps, 2008: a joint guideline from the American Cancer Society, the US Multi-Society Task Force on Colorectal Cancer, and the American College of Radiology. Gastroenterology. 2008; 134(5):1570-95.

18. Malila N, et al. Test, episode, and programme sensitivities of screening for colorectal cancer as a public health policy in Finland: experimental design BMJ. 2008;337:a2261

19. Elsafi SH, et al. The sensitivity, specificity, predictive values, and likelihood ratios of fecal occult blood test for the detection of colorectal cancer in hospital settings. Clin Exp Gastroenterol. 2015;8:279-84.

20. Whitlock EP, et al. Screening for colorectal cancer: a targeted, updated systematic review for the U.S. Preventive Services Task Force. Ann Intern Med. 2008;149(9):638-58.

21. Katsoula A, et al. Diagnostic accuracy of fecal immunochemical test in patients at increased risk for colorectal cancer: a meta-analysis. JAMA Intern Med. 2017;177(8):1110-1118. doi:10.1001/jamainternmed.2017.2309. PubMed PMID: 28628706

22. Imperiale TF, et al. Multitarget stool DNA testing for colorectal-cancer screening. N Engl J Med. 2014;370(14):1287-97.

23. Heigh Rl, et al. Detection of colorectal serrated polyps by stool DNA testing: comparison with fecal immunochemical testing for occult blood (FIT). PLoS One. 2014;9(1):e85659.

24. Lidgard GP, et al. Clinical performance of an automated stool DNA assay for detection of colorectal neoplasia. Clin Gastroenterol Hepatol. 2013;11(10):1313-8.

25. Duffy MJ, et al. Tumour markers in colorectal cancer: European Group on Tumour Markers (EGTM) guidelines for clinical use. Eur J Cancer 2007:43(9):1348-60.

26. Duffy MJ, et al. Clinical utility of biochemical markers in colorectal cancer: European Group on Tumour Markers (EGTM) guidelines. Eur J Cancer. 2003;39(6):718-27.

27. Thomas DS, et al. Evaluation of serum CEA, CYFRA21-1 and CA125 for the early detection of colorectal cancer using longitudinal preclinical samples. Br J Cancer. 2015;113(2):268-74

28. Filiz $\mathrm{Al}$, et al. Persistent high postoperative carcinoembryonic antigen in colorectal cancer patients - is it important? Clinics (Sao Paulo). 2009;64(4):287-94.

29. Duffy MJ. Carcinoembryonic antigen as a marker for colorectal cancer: is it clinically useful? Clin Chem. 2001;47(4):624-30.

30. Su BB, Shi H, Wan J. Role of serum carcinoembryonic antigen in the detection of colorectal cancer before and after surgical resection. World J Gastroenterol. 2012;18(17):2121-6

31. Panel., F.a.D.A.M.a.C.G. FDA executive summary: epigenomics AG Epi proColon. 2014.

32. Yan S, et al. Diagnostic value of methylated septing for colorectal cance screening: a meta-analysis. Med Sci Monit. 2016;22:3409-18.

33. van Rooij, E. and E.N. Olson, MicroRNA therapeutics for cardiovascular disease: opportunities and obstacles. Nat Rev Drug Discov, 2012. 11(11): p. 860-872.

34. Hayes J, Peruzzi PP, Lawler S. MicroRNAs in cancer: biomarkers, function and therapy. Trends Mol Med. 2014;20(8):460-9.
35. Wang QX, et al. Altered MiRNA expression in gastric cancer: a systematic review and meta-analysis. Cell Physiol Biochem. 2015;35(3):933-44.

36. Tilghman SL, et al. Phytoalexins, miRNAs and breast cancer: a review of phytochemical-mediated miRNA regulation in breast cancer. J Health Care Poor Underserved. 2013;24(1 Suppl):36-46.

37. Lodewijk $L$, et al. The value of miRNA in diagnosing thyroid cancer: $a$ systematic review. Cancer Biomark. 2012;11(6):229-38.

38. Srivastava K, Srivastava A. Comprehensive review of genetic association studies and meta-analyses on miRNA polymorphisms and cancer risk. PLoS One. 2012;7(11):e50966.

39. Creemers EE, Tijsen AJ, Pinto YM. Circulating microRNAs: novel biomarkers and extracellular communicators in cardiovascular disease? Circ Res. 2012:110(3):483-95.

40. Larrea $E$, et al. New concepts in cancer biomarkers: circulating miRNAs in liquid biopsies. Int J Mol Sci. 2016;17(5):627.

41. Wahl $P$, et al. Acute effects of different exercise protocols on the circulating vascular microRNAs -16, -21, and -126 in trained subjects. Front Physiol. 2016;7:643.

42. Hsieh $\mathrm{CH}$, et al. Weight-reduction through a low-fat diet causes differential expression of circulating microRNAs in obese C57BL/6 mice. BMC Genomics. 2015;16:699.

43. Ajit SK. Circulating microRNAs as biomarkers, therapeutic targets, and signaling molecules. Sensors (Basel). 2012;12(3):3359-69.

44. Song MY, et al. Identification of serum microRNAs as novel non-invasive biomarkers for early detection of gastric cancer. PLoS One. 2012;7(3):e33608.

45. Brase JC, et al. Serum microRNAs as non-invasive biomarkers for cancer. Mol Cancer. 2010;9:306

46. Chen WY, et al. The potential of plasma miRNAs for diagnosis and risk estimation of colorectal cancer. Int J Clin Exp Pathol. 2015;8(6): 7092-101.

47. Li LP, et al. The diagnostic efficacy and biological effects of microRNA-29b for colon cancer. Technol Cancer Res Treat. 2016;15(6):772-9.

48. Lv ZC, et al. Investigation of microRNA-155 as a serum diagnostic and prognostic biomarker for colorectal cancer. Tumor Biol. 2015;36(3):1619-25.

49. $\mathrm{Ng} \mathrm{L}$, et al. Identification of serum miR-139-3p as a non-invasive biomarker for colorectal cancer. Oncotarget. 2017:8(16):27393-400.

50. Nonaka R, et al. Circulating miR-199a-3p as a novel serum biomarker for colorectal cancer. Oncol Rep. 2014:32(6):2354-8.

51. Pu XX, et al. Circulating miR-221 directly amplified from plasma is a potential diagnostic and prognostic marker of colorectal cancer and is correlated with p53 expression. J Gastroenterol Hepatol. 2010;25(10):1674-80.

52. Shan $L Q$, et al. Diagnostic value of circulating miR-21 for colorectal cancer: a meta-analysis. Cancer Biomarkers. 2015;15(1):47-56.

53. Wang $W$, et al. Circulating miR-210 as a diagnostic and prognostic biomarker for colorectal cancer. Eur J Cancer Care (Engl). 2017;26(4):e12448.

54. Xu CJ, Gu L. The diagnostic effect of serum miR-196b as biomarker in colorectal cancer. Biomed Rep. 2017:6(1):39-45.

55. Yu W, et al. Circulating microRNA-21 as a potential diagnostic marker for colorectal cancer: a meta-analysis. Mol Clin Oncol. 2016:4(2):237-44.

56. Yuan DW, et al. Plasma miR-183 predicts recurrence and prognosis in patients with colorectal cancer. Cancer Biol Ther. 2015;16(2):268-75.

57. Zhi ML, et al. Diagnostic performance of microRNA-29a for colorectal cancer: a meta-analysis. Genet Mol Res. 2015:14(4):18018-25.

58. $\mathrm{Ng} \mathrm{EK}$, et al. Differential expression of microRNAs in plasma of patients with colorectal cancer: a potential marker for colorectal cancer screening. Gut. 2009;58(10):1375-81

59. Huang J, Plass C, Gerhauser C. Cancer chemoprevention by targeting the epigenome. Curr Drug Targets. 2010;12(13):1925-56. Review. PubMed PMID: 21158707.

60. Yang $X$, et al. MicroRNA-92a as a potential biomarker in diagnosis of colorectal cancer: a systematic review and meta-analysis. PLoS One. 2014; 9(2):e88745.

61. Wang J, et al. Identification of a circulating microRNA signature for colorectal cancer detection. PLoS One. 2014;9(4):e87451.

62. Zhang $Y$, et al. Serum microRNA profile in patients with colon adenomas or cancer. BMC Med Genet. 2017:10(1):23.

63. Giraldez MD, et al. Circulating microRNAs as biomarkers of colorectal cancer: results from a genome-wide profiling and validation study. Clin Gastroenterol Hepatol. 2013;11(6):681-8 e3.

64. Yuan $T$, et al. Plasma extracellular RNA profiles in healthy and cancer patients. Sci Rep. 2016;6:19413. 
65. Burch JA, et al. Diagnostic accuracy of faecal occult blood tests used in screening for colorectal cancer: a systematic review. J Med Screen. 2007;14(3):132-7.

66. Wu CW, et al. Detection of miR-92a and miR-21 in stool samples as potential screening biomarkers for colorectal cancer and polyps. Gut. 2012;61(5):739-45.

67. Yau TO, et al. MicroRNA-20a in human faeces as a non-invasive biomarker for colorectal cancer. Oncotarget. 2016;7(2):1559-68.

68. Yau TO, et al. microRNA-221 and microRNA-18a identification in stool as potential biomarkers for the non-invasive diagnosis of colorectal carcinoma. Br J Cancer. 2014;111(9):1765-71.

69. Kalimutho $M$, et al. Differential expression of miR-144* as a novel fecal-based diagnostic marker for colorectal cancer. J Gastroenterol. 2011;46(12):1391-402.

70. Yamada A, et al. Serum miR-21, miR-29a, and miR-125b are promising biomarkers for the early detection of colorectal neoplasia. Clin Cancer Res. 2015:21(18):4234-42.

71. Elshafei A, et al. The expression profiling of serum miR-92a, miR-375, and miR-760 in colorectal cancer: an Egyptian study. Tumour Biol. 2017;39(6):1010428317705765.

72. Krawczyk $P$, et al. Evaluation of miR-506 and miR-4316 expression in early and non-invasive diagnosis of colorectal cancer. Int J Color Dis. 2017:32(7):1057-60.

73. Chang PY, et al. MicroRNA-223 and microRNA-92a in stool and plasma samples act as complementary biomarkers to increase colorectal cancer detection. Oncotarget. 2016;7(9):10663-75.

74. Chen C, et al. Real-time quantification of microRNAs by stem-loop RT-PCR. Nucleic Acids Res. 2005;33(20):e179.

75. Redshaw N, et al. A comparison of miRNA isolation and RT-qPCR technologies and their effects on quantification accuracy and repeatability. BioTechniques. 2013;54(3):155-64

76. Balcells I, Cirera S, Busk PK. Specific and sensitive quantitative RT-PCR of miRNAs with DNA primers. BMC Biotechnol. 2011;11:70

77. Yong FL, Law CW, Wang CW. Potentiality of a triple microRNA classifier: miR-193a-3p, miR-23a and miR-338-5p for early detection of colorectal cancer. BMC Cancer. 2013:13:280.

78. Imaoka H, et al. Circulating microRNA-1290 as a novel diagnostic and prognostic biomarker in human colorectal cancer. Ann Oncol. 2016;27(10):1879-86.

79. Matsumura T, et al. Exosomal microRNA in serum is a novel biomarker of recurrence in human colorectal cancer. Br J Cancer. 2015;113(2):275-81.

80. Phua LC, et al. Global fecal microRNA profiling in the identification of biomarkers for colorectal cancer screening among Asians. Oncol Rep. 2014;32(1):97-104.

81. Draghici $\mathrm{S}$, et al. Reliability and reproducibility issues in DNA microarray measurements. Trends Genet. 2006;22(2):101-9.

82. Sato F, et al. Intra-platform repeatability and inter-platform comparability of microRNA microarray technology. PLoS One. 2009;4(5):e5540.

83. Meyer SU, et al. Profound effect of profiling platform and normalization strategy on detection of differentially expressed microRNAs - a comparative study. PLoS One. 2012;7(6):e38946.

84. Wu D, et al. The use of miRNA microarrays for the analysis of cancer samples with global miRNA decrease. RNA. 2013;19(7):876-88.

85. Pradervand $\mathrm{S}$, et al. Impact of normalization on miRNA microarray expression profiling. RNA. 2009;15(3):493-501.

86. Rao $Y$, et al. A comparison of normalization techniques for microRNA microarray data. Stat Appl Genet Mol Biol. 2008;7(1):Article22.

87. Wang $B, X i$ Y. Challenges for microRNA microarray data analysis. Microarrays (Basel). 2013:2(2):34-50.

88. Margulies $\mathrm{M}$, et al. Genome sequencing in microfabricated high-density picolitre reactors. Nature. 2005;437(7057):376-80.

89. Shao K, et al. Emulsion PCR: a high efficient way of PCR amplification of random DNA libraries in aptamer selection. PLoS One. 2011;6(9):e24910.

90. Ma Z, et al. Isothermal amplification method for next-generation sequencing. Proc Natl Acad Sci U S A. 2013;110(35):14320-3.

91. Goodwin S, McPherson JD, McCombie WR. Coming of age: ten years of next-generation sequencing technologies. Nat Rev Genet. 2016;17(6):333-51.

92. Fuller $\mathrm{CW}$, et al. The challenges of sequencing by synthesis. Nat Biotechnol. 2009;27(11):1013-23.

93. Li J, et al. An NGS workflow blueprint for DNA sequencing data and its application in individualized molecular oncology. Cancer Inform. 2015; 14(Suppl 5):87-107.

94. Ansorge WJ. Next-generation DNA sequencing techniques. New Biotechnol. 2009;25(4):195-203.
95. Chatterjee A, et al. A cross comparison of technologies for the detection of microRNAs in clinical FFPE samples of hepatoblastoma patients. Sci Rep. 2015;5:10438.

96. Malone $\mathrm{JH}$, Oliver B. Microarrays, deep sequencing and the true measure of the transcriptome. BMC Biol. 2011;9:34.

97. Park ST, Kim J. Trends in next-generation sequencing and a new era for whole genome sequencing. Int Neurourol J. 2016;20:76-83.

98. Vychytilova-Faltejskova $\mathrm{P}$, et al. Serum-based microRNA signatures in early diagnosis and prognosis prediction of colon cancer. Carcinogenesis. 2016;37(10):941-50.

99. Ho GYF, et al. Differential expression of circulating microRNAs according to severity of colorectal neoplasia. Transl Res. 2015;166(3):225-32.

100. Zheng G, et al. Serum microRNA panel as biomarkers for early diagnosis of colorectal adenocarcinoma. Br J Cancer. 2014;111(10):1985-92.

101. Zhao $Y$, et al. Isothermal amplification of nucleic acids. Chem Rev. 2015;115(22):12491-545.

102. Jia $\mathrm{H}$, et al. Ultrasensitive detection of microRNAs by exponential isothermal amplification. Angew Chem Int Ed Engl. 2010;49(32):5498-501.

103. Li C, et al. One-step ultrasensitive detection of microRNAs with loop-mediated isothermal amplification (LAMP). Chem Commun (Camb). 2011;47(9):2595-7.

104. Jonstrup SP, Koch J, Kjems J. A microRNA detection system based on padlock probes and rolling circle amplification. RNA. 2006;12(9):1747-52.

105. Tian $Q$, et al. Carbon nanotube enhanced label-free detection of microRNAs based on hairpin probe triggered solid-phase rolling-circle amplification. Nano. 2015;7(3):987-93.

106. Li Y, Liang L, Zhang CY. Isothermally sensitive detection of serum circulating miRNAs for lung cancer diagnosis. Anal Chem. 2013:85(23):11174-9.

107. Sun $Y$, et al. Rapid and direct microRNA quantification by an enzymatic luminescence assay. Anal Biochem. 2012;429(1):11-7.

108. Ge J, et al. A highly sensitive target-primed rolling circle amplification (TPRCA) method for fluorescent in situ hybridization detection of microRNA in tumor cells. Anal Chem. 2014;86(3):1808-15.

109. Drmanac $R$, et al. Human genome sequencing using unchained base reads on self-assembling DNA nanoarrays. Science. 2010;327(5961):78-81.

110. Yin BC, Liu YQ, Ye BC. One-step, multiplexed fluorescence detection of microRNAs based on duplex-specific nuclease signal amplification. J Am Chem Soc. 2012;134(11):5064-7.

111. Pang $Y$, et al. Fe(3)O(4)@Ag magnetic nanoparticles for microRNA capture and duplex-specific nuclease signal amplification based SERS detection in cancer cells. Biosens Bioelectron. 2016;79:574-80.

112. Wang $Q$, et al. Colorimetric detection of sequence-specific microRNA based on duplex-specific nuclease-assisted nanoparticle amplification. Analyst. 2015;140(18):6306-12.

113. Shuai $\mathrm{HL}$, et al. Au nanoparticles/hollow molybdenum disulfide microcubes based biosensor for microRNA-21 detection coupled with duplex-specific nuclease and enzyme signal amplification. Biosens Bioelectron. 2017:89(Pt 2):989-97.

114. Dirks RM, Pierce NA. Triggered amplification by hybridization chain reaction. Proc Natl Acad Sci U S A. 2004;101(43):15275-8.

115. Miao J, et al. A plasmonic colorimetric strategy for visual miRNA detection based on hybridization chain reaction. Sci Rep. 2016;6:32219.

116. Miao X, et al. Sensitive detection of miRNA by using hybridization chain reaction coupled with positively charged gold nanoparticles. Sci Rep. 2016;6:32358

117. Ge Z, et al. Hybridization chain reaction amplification of microRNA detection with a tetrahedral DNA nanostructure-based electrochemical biosensor. Anal Chem. 2014;86(4):2124-30.

118. Yang $L$, et al. Graphene surface-anchored fluorescence sensor for sensitive detection of microRNA coupled with enzyme-free signal amplification of hybridization chain reaction. ACS Appl Mater Interfaces. 2012;4(12):6450-3.

119. Bi S, et al. Triggered and catalyzed self-assembly of hyperbranched DNA structures for logic operations and homogeneous CRET biosensing of microRNA. Chem Commun (Camb). 2016;52(31):5455-8.

120. Cheglakov $Z$, et al. Live cell microRNA imaging using cascade hybridization reaction. J Am Chem Soc. 2015;137(19):6116-9.

121. Cardoso J, et al. Genomic profiling by DNA amplification of laser capture microdissected tissues and array CGH. Nucleic Acids Res. 2004;32(19):e146.

122. Hirsch D, et al. A new whole genome amplification method for studying clonal evolution patterns in malignant colorectal polyps. Genes Chromosomes Cancer. 2012;51(5):490-500. 
123. Aldecoa I, et al. Molecularly determined total tumour load in lymph nodes of stage I-II colon cancer patients correlates with high-risk factors. A multicentre prospective study. Virchows Arch. 2016;469(4):385-94.

124. Rakislova N, et al. Lymph node pooling: a feasible and efficient method of lymph node molecular staging in colorectal carcinoma. J Transl Med. 2017;15(1):14.

125. Persano $S$, et al. Label-free isothermal amplification assay for specific and highly sensitive colorimetric miRNA detection. ACS Omega. 2016;1 (3):448-55.

126. Zhou W, et al. RNA responsive and catalytic self-assembly of DNA nanostructures for highly sensitive fluorescence detection of microRNA from cancer cells. Chem Commun (Camb). 2015;51(92):16494-7.

127. Kim E, et al. Multi-amplified sensing of microRNA by a small DNA fragmentdriven enzymatic cascade reaction. ACS Sens. 2017;2(1):111-8.

128. Feng $C$, et al. Detection of microRNA: a point-of-care testing method based on a $\mathrm{pH}$-responsive and highly efficient isothermal amplification. Anal Chem. 2017:89(12):6631-6.

129. Jiang $H X$, et al. G-quadruplex fluorescent probe-mediated real-time rolling circle amplification strategy for highly sensitive microRNA detection. Anal Chim Acta. 2016;943:114-22.

130. Gong YJ, et al. A unique approach toward near-infrared fluorescent probes for bioimaging with remarkably enhanced contrast. Chem Sci. 2016;7(3):2275-85.

131. Khreis $\mathrm{OM}$, et al. $980 \mathrm{~nm}$ electroluminescence from ytterbium tris (8-hydroxyquinoline). Org Electron. 2001;2(1):45-51.

132. Zhong $Y$, et al. BODIPY chromophores as efficient green light sensitizers for lanthanide-induced near-infrared emission. Dalton Trans. 2011;40:11389-95.

133. Lincheneau C, et al. Recent highlights in the use of lanthanide-directed synthesis of novel supramolecular (luminescent) self-assembly structures such as coordination bundles, helicates and sensors. Aust J Chem. 2011;64(10):1315-26

134. Comby, S. and J.-C.G. Bünzli. Chapter 235 lanthanide near-infrared luminescence in molecular probes and devices. In: Handbook on the physics and chemistry of rare earths. J. Karl A. Gschneidner, J.-C.G. Bünzli, and V.K. Pecharsky, Editors. North Holland: Elsevier; 2007. p. 217-470.

135. Karhunen $U$, et al. Homogeneous detection of avidin based on switchable lanthanide luminescence. Anal Chem. 2011;83(23):9011-6.

136. Karhunen $U$, et al. Luminescence switching by hybridization-directed mixed lanthanide complex formation. Anal Chem. 2010;82(2):751-4.

137. Saneyoshi $H$, Ito $Y$, Abe $H$. Long-lived luminogenic probe for detection of RNA in a crude solution of living bacterial cells. J Am Chem Soc. 2013;135(37):13632-5.

138. Nonat AM, Quinn SJ, Gunnlaugsson T. Mixed f-d coordination complexes as dual visible- and near-infrared-emitting probes for targeting DNA. Inorg Chem. 2009;48(11):4646-8.

139. Mardanya S, et al. Homo- and heterobimetallic ruthenium(II) and osmium(II) complexes based on a pyrene-biimidazolate spacer as efficient DNA-binding probes in the near-infrared domain. Inorg Chem. 2016;55(7):3475-89.

140. Miao $P$, et al. Near-infrared Ag2S quantum dots-based DNA logic gate platform for miRNA diagnostics. Anal Chem. 2016;88(15):7567-73.

141. Shah $P$, et al. In-solution multiplex miRNA detection using DNA-templated silver nanocluster probes. Analyst. 2014;139(9):2158-66.

142. He, H., Near-infrared emitting lanthanide complexes of porphyrin and BODIPY dyes. Coord Chem Rev, 2014. 273-274(0): p. 87-99.

143. $\mathrm{He} \mathrm{H}$, et al. lodized BODIPY as a long wavelength light sensitizer for the nearinfrared emission of ytterbium(III) ion. ChemCommun. 2012;48(13):1886-8.

144. Shavaleev NM, et al. Surprisingly bright near-infrared luminescence and short radiative lifetimes of ytterbium in hetero-binuclear Yb-Na chelates. Inorg Chem. 2009:48(16):7937-46.

145. Kazuki K, Hirotatsu K, Tetsuo N. Functional near-infrared fluorescent probes. Chem Asian J. 2008:3(3):506-15.

146. Borisov SM, Wolfbeis OS. Optical biosensors. Chem Rev. 2008;108(2):423-61.

147. Kobayashi $\mathrm{H}$, et al. New strategies for fluorescent probe design in medical diagnostic imaging. Chem Rev. 2010;110(5):2620-40.

148. Pansare VJ, et al. Review of long-wavelength optical and NIR imaging materials: contrast agents, fluorophores, and multifunctional nano carriers. Chem Mater. 2012;24(5):812-27.

149. Hong GA, AL Dai H. Near-infrared fluorophores for biomedical imaging. Nat Biomed Eng. 2017;1(1):0010.

\section{Submit your next manuscript to BioMed Central and we will help you at every step:}

- We accept pre-submission inquiries

- Our selector tool helps you to find the most relevant journal

- We provide round the clock customer support

- Convenient online submission

- Thorough peer review

- Inclusion in PubMed and all major indexing services

- Maximum visibility for your research

Submit your manuscript at www.biomedcentral.com/submit

) Biomed Central 